\title{
LIABILITY IN AUTONOMOUS VEHICLE ACCIDENTS
}

Nowadays, due to the constant change in the society's expectations regarding transportation, the main area of automotive development is the implementation of autonomous vehicle control functions. While with the gradual introducing and spreading of autonomous functions-even based on the previous experience-the decrease of the number of traffic accidents is expectable, parallel we have to prepare the emergence for the new types of accidents, in which autonomous vehicles will be involved. Needs of follow-up examination and reconstruction of such type accidents makes necessary the general use of the event/accident data recorders in road vehicles and application of the new methods and tools in accident reconstruction and examination of causes of accidents. Examining a road accident it is necessary to investigate the liability issues, as well, what requires new approaches and developing of a uniform practice.

Keywords: autonomous vehicles, driver assistance systems, accident reconstruction, distribution of liability, EDR

\section{Introduction}

Today, society's needs and expectations regarding transportation are undergoing major changes. In order to satisfy the demand of maximizing traffic density, minimizing the number of accidents, optimal utilization of natural resources, minimizing environmental damage, etc. vehicles are necessarily equipped with more and more driver assistant systems, which-in addition to the above-unburden and partially take over the task of the driver. The wide spreading of fully autonomous vehicles will be a determining stage in the development of these systems. Despite the technological progress, occurrence of traffic accidents in the future is unavoidable. Novel causes of accidents are expected in connection to traffic accidents between the manned (driven by a human driver) and autonomous vehicles and between autonomous vehicles. To determine the causes of these types of accidents imposes the novel requirements for accident analysis experts. The development of vehicle systems, especially electronic systems, makes it inevitable to record data related to the operation or malfunctions of those systems, and to make those data accessible for accident analysis experts.

Autonomous vehicles, accident analysis and examination of causes of accidents are the key research areas of our Department.

The aim of this article is to review the current legal and technical situation regarding traffic accidents related data, the expected trend in vehicles development and the EDR systems
(Event Data Recorder, also known as the Crash Data Retrieval) and to make a proposal about the data-set necessary for the effective accident analysis related to autonomous vehicles.

\section{The development of vehicles, the concept of autonomous vehicles}

Nowadays, one of the most important aims of development processes regarding vehicles control is autonomous vehicles. Based on conservative scenarios, the probable value of the ratio of fully autonomous vehicles referring to the number of all vehicles placed on the market as new is $15-20 \%$, with the assumption that the current economic trends continue and there will be no significant changes in the legal environment.

Based on optimistic scenarios, this rate might even be 25-30\%, assuming more flexible safety and technical specifications, increasing state funding and the emergence of the new, innovative market operators.

The SAE (Society of Automotive Engineers) defined the technological levels, which exist between the non-autonomous and fully-autonomous vehicle-systems [1]. These levels and their attributes can be seen in Figure 1.

Cars which are only equipped with the driver assistance systems like lane departure warning (LDW), frontal collision warning (FCW), etc. cannot be considered as autonomous

\footnotetext{
* Krisztian Pinter, Zsolt Szalay, Gabor Vida

Department of Automotive Technologies, Faculty of Transportation Engineering and Vehicle Engineering, Budapest University of Technology and Economics,

E-mail: krisztian.pinter@gjt.bme.hu
} 


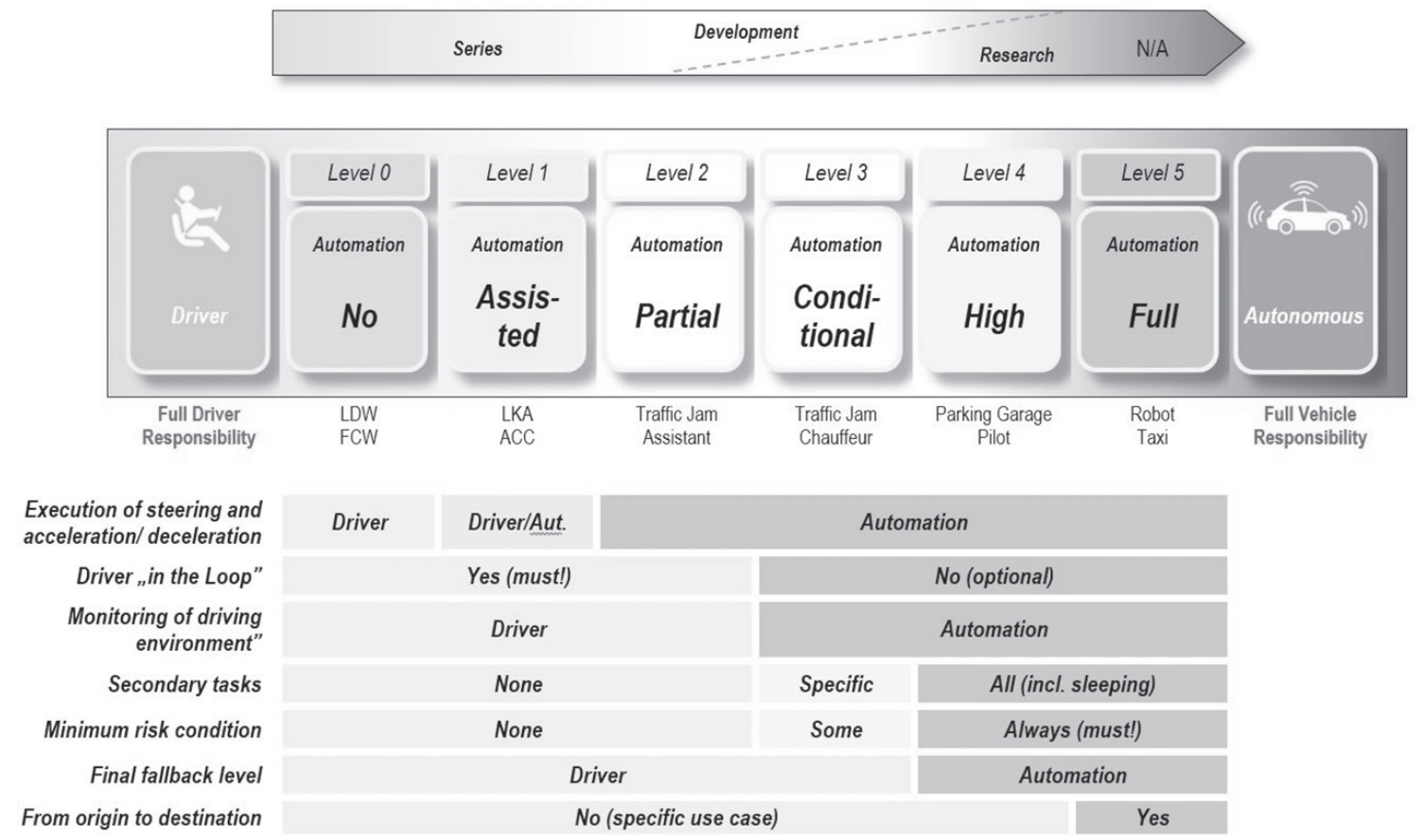

Figure 1 Development process, levels and attributes of the vehicle control

vehicles (Level 0 ). These systems, as their names indicate, only draw the driver's attention to the probable emergency situations, and they cannot intervene in the control of a vehicle.

Systems capable of intervening in the control of vehicles (e.g. by accelerating, braking, steering) only between strictly defined boundary conditions can be found at the first level of automation (Level 1), provided that these systems only proof-correct the parameters set by the driver, in a relatively narrow range. The lane keeping assistant (LKA) and adaptive cruise control (ACC) belong here.

At the second level of automation (Level 2) the vehicle has the ability to control itself-in a relatively small number of situations, between strictly defined boundary conditions-when some accelerating, braking and steering maneuvers are performed by the vehicle itself, for example making one's way along in a traffic jam (Traffic Jam Assistant).

At the levels presented above continuous monitoring and tracking of the traffic environment is the task of the driver and the driver compulsorily takes part in the controlling of the vehicle. Accordingly, in certain traffic conditions the driver has to initiate taking back the control from the vehicle.

At the third level of automation (Level 3) the vehicle can run in fully autonomous mode under conditions nearly identical to the second level. At the same time, when those conditions change, the vehicle initiates to give back control to the driver - in contrast with the automation level at Level 2 (Traffic Jam Chauffeur, Highway Chauffeur)

At the fourth level of automation (Level 4) vehicles can run in fully autonomous mode in broad operating ranges. The control system is always able to take the vehicle into the minimal risk condition in the following cases:

- if the traffic situation justifies it;

- in the case of failure of the autonomous control system of the vehicle;

- when the reaction of the driver is not adequate to the request of the vehicle to intervene (e.g. the driver does not respond to the signs of HMI (Human-Machine Interface), the shaking of the steering wheel).

Nowadays, fourth-level systems include for example the Parking Garage Pilot, which executes parking manoeuvres in fully autonomous mode, or the Highway Pilot, which can run in autonomous mode under highway conditions.

The fundamental difference between the third and fourth level of automation is that while at the third level the final decision regarding the control of the vehicle always has to be taken by the driver in critical operating, at the fourth level this decision is the task of the vehicle.

At the fifth level of automation (Level 5) the vehicle must be able to get from the point of departure to the destination without the intervention of passengers.

In connection to the above, it has to be emphasized that though certain driver assistance systems clearly correspond to certain automation levels, the fact that a vehicle is equipped with a system of a certain level is not sufficient for the whole vehicle to satisfy the requirements of the given level (i.e. the existence of certain assistance systems is a necessary but not sufficient condition for meeting requirements of the given level.) 


\section{Questions of liability regarding highly automated systems}

Nowadays one of the most important aims of development processes regarding vehicles control is autonomous vehicles. Based on conservative scenarios, the probable value of the ratio of fully autonomous vehicles referring to the number of all vehicles placed on the market as new is $15-20 \%$, with the assumption that the current economic trends continue and there will be no significant changes in the legal environment.

With the appearance of highly automated systems, the legal and technical evaluation and assessment of liability require a new approach and interpretation in addition to the usual procedures [2].

The detailed investigation of NHTSA (National Highway Traffic Safety Administration) on an accident that occurred in May 2016 pointed out that even if the ADAS (Advanced driverassistance systems) system presently available, such as Tesla AEB (Automatic Emergency Braking) and its Autopilot systems in that special case, work properly, the driver's continuous supervision and attention is necessary and indispensable [3].

In this specific case, the investigation concluded that the autonomous driver assistance systems of the vehicle functioned properly and the driver had not performed any preventing manoeuver immediately prior to the accident. This shows that at the present level of automation insufficient emphasis is placed on the fact that the driver must perform continuous and complete supervision over the vehicle and its transport-related environment in every situation (partly due to the aggressive marketing strategy of manufacturers) in addition to the proper functioning of the currently available ADAS systems. A good example for this is the term "Autopilot" system used by Tesla, which clearly implies "the ability to drive itself" function for the users. At the same time, the user's manual of the vehicle also contains indications to the limits of the embedded systems and in the active state of the ADAS system the HMI system of the car reminds the driver in text and picture messages to the necessity of being continuous ready to intervene.

However, the accidents having occurred show that those warnings are ignored by the drivers, which is in part most likely due to the fact that the above mentioned pieces of information with contradictory content are not given the same emphasis. Therefore, when developing the ADAS systems of this kind, one of the key tasks is continuously maintaining the driver's attention, as well as recognizing when driver supervision ceases.

Nevertheless, as it was highlighted in the NHTSA report, the ratio of accidents regarding vehicles that are equipped with the Tesla Autopilot systems has decreased for 38 percent as compared to "traditional" (without Autopilot) vehicles [3].

The results mentioned above support Kenneth Leonard's opinion (Director of the Intelligent Transportation Joint Program Office-U.S. DOT), who claims that "...technology alone has the

\begin{tabular}{|l|l|}
\hline Safety concerns / Fail-safe concepts & $76 \%$ \\
\hline Liability issues & $59 \%$ \\
\hline Regulatory mandates / Legal restrictions & $57 \%$ \\
\hline Cyber security & $51 \%$ \\
\hline Social acceptance / Critical perception of self-driving vehicles & $42 \%$ \\
\hline Limitations with regards to infrastructure & $35 \%$ \\
\hline HMI driver attention management & $22 \%$ \\
\hline
\end{tabular}

Figure 2 Expectations of experts from the spread of autonomous driving/vehicles

potential to eliminate 80 percent of the unimpaired collisions" [4].

This is also confirmed by a survey carried out by the experts in the field in 2017, according to which an increase in transport safety and a decrease in the number of accidents is expected due to the appearance of autonomous vehicles in the first place (76\%) (Figure 2).

Therefore, it can be concluded that the expected widespreading of autonomous vehicles can to a great extent contribute to realizing the European Union's ambitious plans regarding the transport safety. They contain the following middle-and long term aims [5]:

- White Book (2001-2010) "The Committee would like to unite the efforts in order to decrease the number of casualties/ deaths for fifty percent during this time period."

- New White Book (2011-2020) "The European Union strives to decrease the number of injuries for fifty percent by 2020 "

- Transport 50 (2020-2050): "Decreasing road accident deaths to almost zero by 2050 has to be achieved."

Parallel with the prospective decrease in the number of roadtraffic accidents, the new types of accidents appear and therefore the three types of accidents can be identified depending on the automation level of the vehicles involved in the accident:

- accidents between conventional - conventional vehicles;

- accidents between conventional - autonomous vehicles (it can be divided into subtypes depending on the degree of automation);

- accidents between autonomous - autonomous vehicles.

The investigation of these new types of conflicts raises new questions in connection with the investigation of liability in addition to the novel set of expert and technical instruments [6].

When considering a vehicle involved in a road-traffic accident, the impact of the following entities concerned can be examined when investigating the accident liability: vehicle driver, keeper, producer (OEM-Original Equipment Manufacturer), system vendor (TIER1), operator of transport infrastructure [7].

With regard to the above, it has to be taken into consideration that the current basis for the legal regulation of road transport is the 1968 Vienna Convention on Road Traffic. It clearly states that there must be a vehicle driver present in all the moving vehicles 
or combination of vehicles, who has to be able to continuously control their vehicle.

Since the Vienna Convention on Road Traffic has not been ratified by each and every country, the current possibilities for testing and operating the self-driving cars vary worldwide.

The differing regulation provides opportunity for the vehicle producers and system vendors to perform road tests already at the current state of the development. At the same time, in the USA, for instance, it was the speaker of the coalition for self-driving cars who indicated that they consider it unfortunate that the individual states regulate the testing and operating conditions of autonomous vehicles on a state level, since a coherent regulation on a federal level would be significantly more efficient.

Until January of 2017, in the US 33 states have introduced autonomous vehicle legislation, and there are some states, where this regulations allow testing self-driving car on public roads, even without human driver on the board. In April 2016 the Amsterdam Declaration has been signed by all $28 \mathrm{EU}$ member states: this declaration is to regulate and unify the implementation of autonomous-driving vehicles on European roads, including to make suitable legal environment for testing the self-driving cars on public roads in Europe. For example, in May 2017, Germany has passed a law that gives auto companies permission to test their self-driving cars on public roads: the most important safety conditions of it is a presence of a human driver behind the wheel at all times with the ability to take over from the car's AI (Artificial Intelligence) when needed, and that all the self-driving cars must have a black box-like device that can record when the $\mathrm{AI}$ is in control and when a human is doing the driving. If at the time of the accident-by the data of the EDR-the human was the driver of the car, he or she may be responsible for the accident, in other cases, the car manufacturer will be held responsible for an accident caused by the AI.

Taking into consideration the current regulation regarding conventional vehicles, liability lies mainly with the driver/keeper. However, depending on the degree of automation, liability shifts significantly towards the manufacturer / the system vendor / the operator of traffic infrastructure (Figure 3).

The reasons for these are the following:

- if the vehicle does not have a driver, at the final level of automation, he/she cannot be rendered liable;

- with the appearance of the functions for autonomous vehicles, system vendors supply a complete system, while the car manufacturer "only" a systems integration. In consequence, liability due to the improper operation of the vehicle can be shared between the two parties. It also follows that while regarding conventional vehicles manufacturer liability is only considered in connection with structural defects, regarding autonomous vehicles liability of the logic (= "the driver") controlling the vehicle, i.e. liability of the manufacturer/ vendor can be determinative. In this respect, it is to be investigated whether the programmed decision algorithm complies with the effective legal requirements (perhaps generally accepted moral principles) [8].

autonomous vehicles can get different pieces of information about the condition of transport infrastructure, or even its elements with their environment sensor systems or during the V2I (Vehicle-to-Infrastructure) communication. If these pieces of information are defective, liability of the systems creating and forwarding the information (its operator) is to be investigated [9].

Regarding the liability questions it is important to emphasize that autonomous vehicles-as their name suggests-are not only capable of executing pre-programmed operations, but under specific circumstances, interacting with their environment, they can also make unpredicted, independent decisions by means of their autonomous functions. In that sense, an autonomous vehicle cannot be clearly considered as a tool in the hands of its manufacturer, owner or user, so the current liability regulations (e.g. product liability) are not completely applicable for dealing with damages caused by operating the vehicle [10]. It is necessary to examine, from a legal point of view, as what kind of an entity (natural, legal, maybe animal or object) an autonomous vehicle, with possible cognitive functions, can be considered or whether it is justifiable to create an independent category for autonomous vehicles and robots. As regards to autonomous vehicles, it causes problems that based on the current legal regulations, it is not possible to identify clearly the person responsible for the damage caused by the vehicle. In that respect, also considering current legal practice, it seems appropriate to apply a kind of objective liability regarding autonomous vehicles.

According to this, it is sufficient to prove only the fact that there is a cause-and-effect relation between the actions of the autonomous vehicle causing damage and the damage suffered by the person harmed. When identifying people with liability and assigning liability, it can be a general approach that the parties' liability has to be proportional to the actual level of the instructions given to the vehicle and the autonomy level of the vehicle.

Finding the answers to the above mentioned questions, which partly arise with the spreading of autonomous vehicles, requires the novel expert tools and methods. When creating those methods, the followings must also be taken into consideration: potential special accident scenarios to be expected in the irregular operation of autonomous vehicles, the elements of intelligent traffic environment. Since it is possible, already at the lower levels of automation that - under specific operational circumstances - the logic controlling the vehicle makes control decisions independently, it is essential to use an event data recorder (EDR). Such data recorders, i.e. data recording functions, are already available in current vehicles, either as manufactured (for passenger cars it is obligatory in the USA since 2014, its data content is recorded in detail by a 49 CFR Part 563 [11] or built in afterwards. 


\section{General sharing/distribution of accident liability depending on automation level of the vehicle}

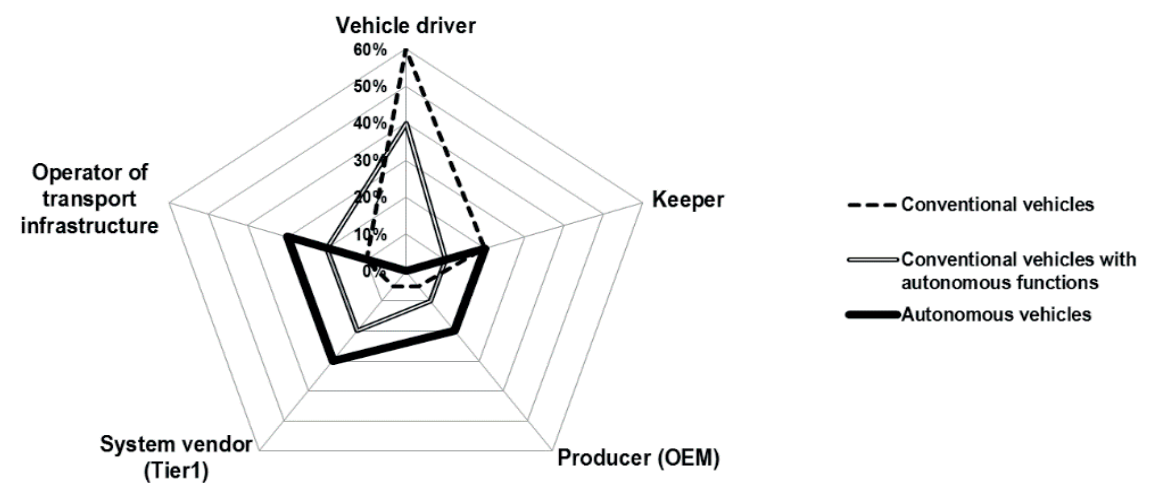

Figure 3 General sharing/distribution of accident liability depending on automation level of the vehicle

During the follow-up accident reconstruction, accidents of autonomous vehicles, especially those running without a driver, can be treated similarly to accidents where there is lack of trace. Therefore, in the case of accidents involving autonomous vehicles, the value of operational and environmental parameters, attributes of vehicle dynamics stored prior to, during and following the accident, in the various control units or perhaps dedicated devices of the vehicles involved in the accident, is particularly enhanced, since they can provide objective clues for accident reconstruction.

However, in addition to the data content stored on current event data recorders, it is also necessary to record the following:

- Who was controlling the vehicle at the time of the accident? Was it the control realising the autonomous function or the driver?

- Was the driver watchful prior to the accident: did he monitor the traffic environment? It is necessary to investigate this even if the vehicle was running in a self-driving mode.

- Which systems were operating / intervened in the control of the vehicle at the time of the accident? It is possible, even besides human driving, that a certain active safety system or driver assistance system has come into action immediately preceding the accident.

- Which vehicles or which elements of the traffic infrastructure the vehicle communicated with prior to the accident and what kind of information did it receive from those or transmit to those? [12]

- If the control of the vehicle was given over (from the driver to the vehicle or vice versa) immediately before the accident (approximately in the 3 minutes preceding), in what manner the handover and takeover took place, when exactly it happened compared to the accident and who initiated the handover.

When developing and defining the extended EDR functions, which can be used effectively even in autonomous vehicles, the extent to which the vehicle is autonomous has to be taken into consideration. Accordingly, what functions or sensors supplying information is the vehicle equipped with: it is necessary to record the active/passive status of these systems, or rather those of their sensors, their performance, as well as the signals of the sensors.

\section{Conclusion}

With the probable appearance of autonomous vehicles, the novel types of accident causes are expected to emerge regarding accidents between the human-driven and autonomous vehicles or between autonomous-autonomous vehicles. The novel accident causes will also result in the novel liability conditions. In connection with the technical and the follow-up enforcement evaluation of those the following are to be expected:

- It becomes necessary to expand the data content of the EDR systems recording certain technical parameters of the process of the accident.

- Depending on the automation degree of the vehicles, liability shifts significantly from the driver / keeper towards manufacturer / system supplier / operator of the traffic infrastructure.

- Co-operation of the legal and technical fields will be needed, not only following the irregular operation of the vehicle, but already during the development of automation functions.

\section{List of Abbreviations}

EDR - Event Data Recorder

SAE - Society of Automotive Engineers

LDW - Lane Departure Warning

FCW - Frontal Collision Warning 
LKA - Lane Keeping Assistant

HMI - Human-Machine Interface

NHTSA - National Highway Traffic Safety Administration

ADAS - Advanced Driver-Assistance Systems
AEB - Automatic Emergency Braking

OEM - Original Equipment Manufacturer

AI - Artificial Intelligence

V2I - Vehicle-to-Infrastructure

\section{References}

[1] SAE STANDARD J3016: Taxonomy and Definitions for Terms Related to On-Road Motor Vehicle Automated Driving Systems. 2014.

[2] GRUEL, W., STANFORD J. M.: Assessing the Long-Term Effects of Autonomous Vehicles: A Speculative Approach. European Transport Conference, 2015.

[3] HABIB, K., RIDELLA, S., QUANDT, J.: The Automatic Emergency Braking (AEB) or Autopilot Systems May Not Function as Designed, Increasing the Risk of a Crash. NHTSA ODI Resume (PE 16-007), 2017.

[4] KENNETH, L.: Transforming Transportation with Autonomous Vehicles and the Sharing Economy: Are We Ready? Brookings Institution, Washington DC, 2016.

[5] European Commission, European Road Safety Observatory: Annual Accident Report 2016. 2017.

[6] VIERECKL, R., AHLEMANN, D., KOSTER, A., JURSCH, A.: Connected Car Study 2015: Racing Ahead with Autonomous Cars and Digital Innovation [online]. 2015. Available: http://www.strategyand.pwc.com/reports/connected-car-2015-study.

[7] European Commission: Evaluation of the Directive 85/374/EEC Concerning Liability for Defective Products. Evaluation and Fitness Check Roadmap. 2016.

[8] CERKA, P., GRIGIENÉ, J., SIRBIKYTÉ, G.: Liability for damages caused by artificial intelligence. Computer Law \& Security Review. Volume 31, Issue 3, Pages 376-389, 2015.

[9] TETTAMANTI, T., VARGA, I., SZALAY, Z.: Impacts of Autonomous Cars from a Traffic Engineering Perspective. Period. Polytech. Transp. Eng., 44(4), 244-250, 2016.

[10] European Commission: GEAR 2030. Discussion paper, 2016.

[11] NHTSA CDR Ruling 49 CFR Part 563.

[12] SACH, J.: Vehicular Communications Via cellular Networks. Ericsson Research, 2015. 